\title{
The Phase-Space Noncommutativity Effect on the Large and Small Wave-Function Components Approach at Dirac Equation
}

\author{
Ilyas Haouam \\ Département de Physique, Faculté des Sciences, University des Frères Mentouri Constantine 1, Constantine, Algeria \\ Email: ilyashaouam@live.fr
}

How to cite this paper: Haouam, I. (2018) The Phase-Space Noncommutativity Effect on the Large and Small Wave-Function Components Approach at Dirac Equation. Open Access Library Journal, 5: e4108. https://doi.org/10.4236/oalib.1104108

Received: November 1, 2017

Accepted: September 10, 2018

Published: September 13, 2018

Copyright $\odot 2018$ by author and Open Access Library Inc.

This work is licensed under the Creative Commons Attribution International License (CC BY 4.0).

http://creativecommons.org/licenses/by/4.0/

\section{(c) (i) Open Access}

\begin{abstract}
By the large and small wave-function components approach we achieved the nonrelativistic limit of the Dirac equation in interaction with an electromagnetic potential in noncommutative phase-space, and we tested the effect of the phase-space noncommutativity on it, knowing that the nonrelativistic limit of the Dirac equation gives the Schrödinger-Pauli equation.
\end{abstract}

\section{Subject Areas}

Applied Physics, Mathematical Analysis, Modern Physics, Quantum Mechanics, Theoretical Physics

\section{Keywords}

Schrödinger-Pauli Equation, Noncommutative Nonrelativistic Limit, Noncommutativity of Phase-Space, Noncommutative Dirac Equation, Moyal Product, Bopp-Shift Transformation

\section{Introduction}

In the last few years there has been much interests in the study of physics in non-commutative space, knowing that the study of noncommutative geometry has a long history [1] [2], the studies of noncommutativity in phase-space and their involvement for quantum field theories play an important role in various fields of physics especially in the theory of strings, and in the matrix model of M-theory [3], also in the description of quantum gravity.

The common method for studying the noncommutativity of quantum mechanics (NCQM) is the correspondence between commutative space and the noncommutative space using the method of translation which known as Bopp-shift, 
or using the Moyal star product [4] [5] [6] [7].

In the quantum-mechanical description of particles, there are various relativistic or non-relativistic wave equations as the usual Schrödinger equation applies to the spin-0 particles in the non-relativistic domain, and the Klein-Gordon equation is the relativistic equation appropriate for spin-0 particles [8] [9] [10], and in regards to the spin-1/2 particles are governed by the relativistic Dirac.

To move from the relativistic quantum mechanics toward the nonrelativistic one it is very necessary to pass by the nonrelativistic limit, which is to transform the physical information under the condition $v \ll c$, we speak of the nonrelativistic limit for low speeds in front of the speed of the light or for the regime of weak or low energy in front of the mass energy $\frac{v}{c} \approx \frac{p c}{m c^{2}} \ll 1$, the nonrelativistic limit can be achieved through various ways, the most important ways are the Foldy-Wouthuysen transformation (it is only applicable to weak fields) [11] [12], and the Douglas-Kroll-Hell transformation [13] [14] [15], this canonical transformation is an unitary transformation allows separating (block-diagonalize) Dirac hamiltonian into two parts, one part describes electrons, while the other gives rise to negative energy states, which are the so-called positronic states, and the classical approach which is the large and small wave function components approach [16] [17], in this work we investigate the nonrelativistic limit of the Dirac equation according to the large and small wave function components approach to derive directly the Schrödinger-Pauli equation [18] [19] [20], but in the case of particles with spin 1 or higher, only relativistic equations are usually considered [21], noting that the uses of the Schrödinger-Pauli equation represented on the study of the fine structure of the hydrogen atom, and various scattering problems knowing that it does not consider the spin of the particles in the studies, but it can be introduced by assuming the presence of an electromagnetic field in the Dirac equation before the extraction of the nonrelativistic equation, which describes the interaction of a spin 1/2 particle with the external electromagnetic field. It correctly predicts the spin of the particle and the gyromagnetic ratio, in fact the examples of using and applying Schrödinger-Pauli equation are many and we can not all mention them.

\section{Length-Momentum Noncommutativity}

At string scales (very small scales) the space does not commute anymore, so that we consider the operators of coordinates and momentum in the noncommutative phase-space $x_{i}^{n c}$ and $p_{i}^{n c}$ respectivly, then considering a noncommutative algebra satisfying the commutation relations

$$
\begin{aligned}
& {\left[x_{i}^{n c}, x_{j}^{n c}\right]=i \Theta_{i j}} \\
& {\left[p_{i}^{n c}, p_{j}^{n c}\right]=i \bar{\Theta}_{i j} \quad(i, j=1,2),} \\
& {\left[x_{i}^{n c}, p_{j}^{n c}\right]=i \hbar^{e f f} \delta_{i j}}
\end{aligned}
$$

taking into account the effective Plank constant 


$$
\hbar^{e f f}=\hbar\left(1+\frac{\Theta \cdot \bar{\Theta}}{4 \hbar^{2}}\right)
$$

where $\Theta_{i j}=\epsilon_{i j k} \Theta_{k}, \quad \Theta_{k}=(0,0, \Theta), \quad \bar{\Theta}_{i j}=\epsilon_{i j k} \bar{\Theta}_{k}, \quad \bar{\Theta}_{k}=(0,0, \bar{\Theta}), \quad \Theta, \bar{\Theta}$ are antisymmetric constant matrices (noncommutative parameters) with the dimension of (lenght) $)^{2}$ and (momentum) $)^{2}$ respectively.

The mapping between the noncommutative phase space and the commutative one doing through the Bopp-Shift linear transformations [22] [23].

$$
\begin{array}{ll}
x^{n c}=x-\frac{1}{2 \hbar} \Theta p_{y} & y^{n c}=y+\frac{1}{2 \hbar} \Theta p_{x} \\
p_{x}^{n c}=p_{x}+\frac{1}{2 \hbar} \bar{\Theta} y & p_{y}^{n c}=p_{y}-\frac{1}{2 \hbar} \bar{\Theta} x
\end{array}
$$

vanishing the noncommutative parameters the system will reduce to the commutative one.

With another method the noncommutativity in space can be realized using the Moyal product ( $\star$-product) [24] [25] [26].

$$
\begin{aligned}
& (f \star g)(x)=\exp \left[\frac{i}{2} \Theta_{a b} \partial_{x_{a}} \partial_{x_{b}}\right] f\left(x_{a}\right) g\left(x_{b}\right) \\
& =f(x) g(x)+\sum_{n=1}\left(\frac{1}{n !}\right)\left(\frac{i}{2}\right)^{n} \Theta^{a_{1} b_{1}} \cdots \Theta^{a_{k} b_{k}} \partial_{a_{1}} \cdots \partial_{a_{k}} f(x) \partial_{b_{1}} \cdots \partial_{b_{k}} g(x),
\end{aligned}
$$

in other term the noncommutativity information is encoded in the Star product

$$
(A, \star)=\left(A^{n c}, .\right)
$$

\section{Nonrelativistic Limit of the Noncommutative Dirac Equation}

\subsection{Noncommutative Dirac Equation}

Starting with the Dirac equation in the noncommutative phase space [27] [28]

$$
H(x, p) \star \psi(x)=H\left(x^{n c}, p^{n c}\right) \cdot \psi\left(x^{n c}\right)=E \psi,
$$

where the Dirac equation in interaction with electromagnetic four-potential $\left\{A_{\mu}=\left(A_{0}, A_{i}(x)\right)\right\}$ in commutative phase space is

$$
\left\{c \hat{\alpha}_{i}\left(\hat{p}_{i}-\frac{e}{c} A_{i}(x)\right)+e A_{0}(x)+\hat{\beta} m c^{2}\right\} \psi=E \psi,
$$

where the momentum $\hat{p}_{i}$ is giving by $\hat{p}_{i}=i \hbar \nabla_{i}$, and the matrices $\hat{\alpha}_{i}$ and $\hat{\beta}$ satisfy the anticommutation relations

$$
\left\{\hat{\alpha}_{i}, \hat{\alpha}_{j}\right\}=2 \delta_{i j},\left\{\hat{\alpha}_{i}, \hat{\beta}\right\}=0, \hat{\alpha}_{i}^{2}=\hat{\beta}^{2}=1 .
$$

Using the Equation (4), we achieve the noncommutativity in space $\left\{x \rightarrow x^{n c}\right\}$, then the Dirac Equation (7) transforms to Equation (6) as

$$
\left\{c \hat{\alpha}_{i}\left(\hat{p}_{i}-\frac{e}{c} A_{i}(x)\right)+e A_{0}(x)+\hat{\beta} m c^{2}\right\} \star \psi(x)=E \psi_{n c},
$$

as $A(x)=h x$ for that the derivation in Equation (4) will automatically stop in 
the first order

$$
(f \star g)(x)=\frac{i}{2} \Theta^{a b} \partial_{a} f \partial_{b} g+\mathrm{O}\left(\Theta^{2}\right),
$$

it means that terms upper than the first order will vanish, the Equation (9) transforms to

$$
\begin{aligned}
& (H \star \psi)(x) \\
& =H\left(x, p^{n c}\right) \psi(x)+\frac{i}{2} \Theta_{a b} \partial_{a}\left(c \hat{\alpha}_{i}\left(\hat{p}_{i}-\frac{e}{c} A_{i}(x)\right)+e A_{0}(x)+\hat{\beta} m c^{2}\right) \partial_{b} \psi(x)(11) \\
& =E \psi_{n c},
\end{aligned}
$$

as $\partial_{a}\left(c \hat{\alpha}_{i} \hat{p}_{i}\right)=\partial_{a}\left(\hat{\beta} m c^{2}\right)=0$, Equation (20) becomes

$$
H\left(x, p^{n c}\right) \psi(x)-\frac{i e}{2} \Theta_{a b} \partial_{a}\left(c \hat{\alpha}_{i} A_{i}(x)+e A_{0}(x)\right) \partial_{b} \psi(x)=E \psi_{n c},
$$

after there we achieve the noncommutativity in phase $\left\{p \rightarrow p^{n c}\right\}$ using the Equation (3) for finding the entire noncommutative phase space Dirac equation,

$$
\begin{aligned}
& H\left(x, p^{n c}\right) \star \psi(x) \\
&=\left\{c \hat{\alpha}_{i}\left(p_{i}+\frac{1}{2 \hbar} \bar{\Theta}_{i j} x_{j}-\frac{e}{c} A_{i}(x)\right)+e A_{0}(x)+\hat{\beta} m c^{2}\right. \\
&\left.-\frac{i e}{2} \Theta_{a b} \partial_{a}\left(c \hat{\alpha}_{i} A_{i}(x)+e A_{0}(x)\right) \partial_{b}\right\} \psi(x) \\
&= E \psi_{n c},
\end{aligned}
$$

we rewrite, the Equation (13) in more compact forme (see Appendix):

$$
\begin{aligned}
& H_{n c} \star \psi_{n c} \\
& =\left\{c \overrightarrow{\hat{\alpha}}\left(\vec{p}-\frac{e}{c} \vec{A}\right)+e A_{0}+\hat{\beta} m c^{2}+\frac{c}{\hbar}(\overrightarrow{\hat{\alpha}} \times \vec{x}) \cdot \vec{\Theta}+\frac{e}{\hbar}\left(\vec{\nabla}\left(\overrightarrow{\hat{\alpha}} \vec{A}-A_{0}\right) \times \vec{p}\right) \cdot \vec{\Theta}\right\} \psi_{n c} \\
& =E \psi_{n c} .
\end{aligned}
$$

\subsection{Large and Small Wave-Function Components Approach}

It is possible to define the nonrelativistic limit of the Dirac equation, using several ways, including that there is the Douglas-Kroll-Hell approach, it used mostly as part of relativistic quantum chemistry, and the Foldy-Wouthuysen transformation, which are both canonical transformation, and the method of development in power of $\hbar$ [29], and the classical approach, the latter one depends on the upper two components of the Dirac wave-function $\psi$ in the standard representation are much larger than the lower two components, using this property we can derive simply the Schrödinger-Pauli equation.

To define the nonrelativistic limit of the phase-space noncommutative Dirac equation we should firstly study the case of an electron at rest, so that without the electromagnetic interaction $\left\{p \psi=0, A^{\mu}=0\right\}$, Equation (14) becomes

$$
H_{n c} \psi_{n c}=\left\{\hat{\beta} m_{0} c^{2}+\frac{c}{\hbar}(\overrightarrow{\hat{\alpha}} \times \vec{x}) \cdot \overrightarrow{\vec{\Theta}}\right\} \psi_{n c}=E \psi_{n c} .
$$

This system of equations is simply solved, and leads to the following 
four-solutions

$$
\begin{aligned}
& \psi_{n c}^{1}=\left(\begin{array}{l}
1 \\
0 \\
0 \\
0
\end{array}\right) \mathrm{e}^{-\frac{i}{\hbar}\left(m_{0} c^{2}+\frac{c}{\hbar}(\overrightarrow{\hat{\alpha}} \times \vec{x}) \cdot \overrightarrow{\bar{\Theta}}\right) t} \psi_{n c}^{2}=\left(\begin{array}{l}
0 \\
1 \\
0 \\
0
\end{array}\right) \mathrm{e}^{-\frac{i}{\hbar}\left(m_{0} c^{2}+\frac{c}{\hbar}(\overrightarrow{\hat{\alpha}} \times \vec{x}) \cdot \overrightarrow{\bar{\theta}}\right) t} \\
& \psi_{n c}^{3}=\left(\begin{array}{l}
0 \\
0 \\
1 \\
0
\end{array}\right) \mathrm{e}^{+\frac{i}{\hbar}\left(m_{0} c^{2}+\frac{c}{\hbar}(\overrightarrow{\hat{\alpha}} \times \vec{x}) \cdot \overrightarrow{\bar{\theta}}\right) t} \psi_{n c}^{4}=\left(\begin{array}{l}
0 \\
0 \\
0 \\
1
\end{array}\right) \mathrm{e}^{+\frac{i}{\hbar}\left(m_{0} c^{2}+\frac{c}{\hbar}(\overrightarrow{\hat{\alpha}} \times \vec{x}) \cdot \overrightarrow{\bar{\theta}}\right) t},
\end{aligned}
$$

$\psi_{n c}^{1}$ and $\psi_{n c}^{2}$ correspond to the positive energy value and $\psi_{n c}^{3}, \psi_{n c}^{4}$ to the negative one.

At first, therefore we restrict ourselves to solutions of positive energy. In order to show that the Dirac equation reproduces the two component Pauli equation in the nonrelativistic limit.

The nonrelativistic limit of the Equation (14) can be most efficiently studied in the representation

$$
\psi_{n c}=\left(\begin{array}{c}
\tilde{\phi}_{n c} \\
\tilde{\chi}_{n c}
\end{array}\right)
$$

where the four-component spinor $\psi_{n c}$ is decomposed into two-two component spinors $\tilde{\phi}_{n c}$ and $\tilde{\chi}_{n c}$, with $\left\{\vec{p}-\frac{e}{c} \vec{A} \rightarrow \vec{\Pi}\right\}$, the Dirac Equation (14) becomes

$$
\begin{aligned}
i \hbar \frac{\partial}{\partial t}\left(\begin{array}{l}
\tilde{\phi}_{n c} \\
\tilde{\chi}_{n c}
\end{array}\right)= & c \overrightarrow{\hat{\alpha}} \vec{\Pi}\left(\begin{array}{l}
\tilde{\phi}_{n c} \\
\tilde{\chi}_{n c}
\end{array}\right)+e A_{0}\left(\begin{array}{l}
\tilde{\phi}_{n c} \\
\tilde{\chi}_{n c}
\end{array}\right)+\hat{\beta} m_{0} c^{2}\left(\begin{array}{l}
\tilde{\phi}_{n c} \\
\tilde{\chi}_{n c}
\end{array}\right) \\
& +\frac{c}{\hbar}(\overrightarrow{\hat{\alpha}} \times \vec{x}) \cdot \overrightarrow{\hat{\Theta}}\left(\begin{array}{l}
\tilde{\phi}_{n c} \\
\tilde{\chi}_{n c}
\end{array}\right)+\frac{e}{\hbar}\left(\vec{\nabla}\left(\overrightarrow{\hat{\alpha}} \vec{A}-A_{0}\right) \times \vec{p}\right) \cdot \vec{\Theta}\left(\begin{array}{l}
\tilde{\phi}_{n c} \\
\tilde{\chi}_{n c}
\end{array}\right),
\end{aligned}
$$

according to the Dirac matrices

$$
\overrightarrow{\hat{\alpha}}=\left(\begin{array}{cc}
0 & \hat{\vec{\sigma}} \\
\hat{\vec{\sigma}} & 0
\end{array}\right), \hat{\beta}=\left(\begin{array}{cc}
1 & 0 \\
0 & -1
\end{array}\right),
$$

and setting $\Xi_{\bar{\Theta}}=(\overrightarrow{\hat{\alpha}} \times \vec{x}) \cdot \vec{\Theta}$, and $\Xi_{\Theta}=\left(\vec{\nabla}\left(\overrightarrow{\hat{\alpha}} \vec{A}-A_{0}\right) \times \vec{p}\right) \cdot \vec{\Theta}$ it comes

$$
\begin{aligned}
i \hbar \frac{\partial}{\partial t}\left(\begin{array}{l}
\tilde{\phi}_{n c} \\
\tilde{\chi}_{n c}
\end{array}\right)= & \left(\begin{array}{l}
c \hat{\sigma} \mathbb{\Pi} \tilde{\phi}_{n c} \\
c \hat{\sigma} \mathbb{\Pi} \tilde{\chi}_{n c}
\end{array}\right)+e A_{0}\left(\begin{array}{l}
\tilde{\phi}_{n c} \\
\tilde{\chi}_{n c}
\end{array}\right)+m_{0} c^{2}\left(\begin{array}{c}
\tilde{\phi}_{n c} \\
-\tilde{\chi}_{n c}
\end{array}\right) \\
& +\frac{c}{\hbar} \Xi_{\bar{\Theta}}\left(\begin{array}{l}
\tilde{\phi}_{n c} \\
\tilde{\chi}_{n c}
\end{array}\right)+\frac{e}{\hbar} \Xi_{\Theta}\left(\begin{array}{l}
\tilde{\phi}_{n c} \\
\tilde{\chi}_{n c}
\end{array}\right),
\end{aligned}
$$

if the rest energy $m_{0} c^{2}$, as the largest occurring energy, is additionally separated by $\left(\begin{array}{l}\tilde{\phi}_{n c} \\ \tilde{\chi}_{n c}\end{array}\right)=\left(\begin{array}{l}\phi_{n c} \\ \chi_{n c}\end{array}\right) \mathrm{e}^{-\frac{i}{\hbar}\left(m_{0} c^{2}+\frac{c}{\hbar} \Xi^{-}\right) t}$, the Equation (18) takes the form

$$
i \hbar \frac{\partial}{\partial t}\left(\begin{array}{l}
\phi_{n c} \\
\chi_{n c}
\end{array}\right)=\left(\begin{array}{c}
c \hat{\vec{\sigma}} \mathbb{\Pi} \chi_{n c} \\
c \hat{\vec{\sigma}} \phi_{n c}
\end{array}\right)+e A_{0}\left(\begin{array}{l}
\phi_{n c} \\
\chi_{n c}
\end{array}\right)-2 m_{0} c^{2}\left(\begin{array}{c}
0 \\
\chi_{n c}
\end{array}\right)+\frac{e}{\hbar} \Xi_{\Theta}\left(\begin{array}{l}
\phi_{n c} \\
\chi_{n c}
\end{array}\right),
$$


firstly considering the lower of the above equation. Using the slow-time dependence $E_{0} \gg i \hbar \frac{\partial}{\partial t}$, and the weak coupling of the electromagnetic potential $E_{0} \gg e A_{0}$ approach, which means that the kinetic energy as well as the potential energy are small compared to the rest energy, by another term the transition to the nonrelativistic limit is realized by assuming that the momentum is small compared to the characteristic quantity $\mathrm{mc}$ and that the Coulomb interaction energy is weak compared to the mass energy, so that the Equation (21) goes to

$$
\left(\begin{array}{l}
c \hat{\sigma} \vec{\Pi} \chi_{n c} \\
c \hat{\vec{\sigma}} \phi_{n c}
\end{array}\right)-2 m_{0} c^{2}\left(\begin{array}{c}
0 \\
\chi_{n c}
\end{array}\right)+\frac{e}{\hbar} \Xi_{\Theta}\left(\begin{array}{l}
\phi_{n c} \\
\chi_{n c}
\end{array}\right)=0,
$$

using the second equation of the above system Equation (22) then we obtain

$$
\chi_{n c}=\frac{c \hat{\sigma} \vec{\Pi}}{2 m_{0} c^{2}-\frac{e}{\hbar} \Xi_{\Theta}} \phi_{n c},
$$

where $\chi_{n c}$ represent the small component of the wave function $\psi_{n c}$. Insertion of Equation (23) into the first equation of Equation (21) results in a nonrelativistic wave function for $\phi_{n c}$

$$
i \hbar \frac{\partial}{\partial t} \phi_{n c}=\frac{(\hat{\vec{\sigma}} \vec{\Pi})(\hat{\sigma} \vec{\Pi})}{2 m_{0}-\frac{e}{\hbar c^{2}} \Xi_{\Theta}} \phi_{n c}+e A_{0} \phi_{n c}+\frac{e}{\hbar} \Xi_{\Theta} \phi_{n c},
$$

with the help of

$$
(\hat{\vec{\sigma}} \hat{\vec{A}})(\hat{\vec{\sigma}} \hat{\vec{B}})=\hat{\vec{A}} \hat{\vec{B}}+i \hat{\vec{\sigma}} \hat{\vec{A}} \times \hat{\vec{B}}
$$

Finally, the Equation (24) becomes

$$
i \hbar \frac{\partial}{\partial t} \phi_{n c}=\left\{\frac{\left(\vec{p}-\frac{e}{c} \vec{A}\right)^{2}}{2 m_{0}-\frac{e}{\hbar c^{2}} \Xi_{\Theta}}-\frac{e \hbar(\hat{\vec{\sigma}} \vec{B})}{c\left(2 m_{0}-\frac{e}{\hbar c^{2}} \Xi_{\Theta}\right)}+e A_{0}+\frac{e}{\hbar} \Xi_{\Theta}\right\} \phi_{n c} .
$$

This is as it should be the noncommutative phase-space Schrödinger-Pauli equation.

For $\Theta=0 \Rightarrow \Xi_{\Theta}=0$, the Equation (26) returns to that of usual SchrödingerPauli equation [30] [31].

\subsection{Gyromagnetic Factor of the Electron ( $g=2)$}

According to $\vec{B}=\vec{\nabla} \times \vec{B}, \vec{A}=\frac{1}{2} \vec{B} \times \vec{x}$, we have

$$
\left(\vec{p}-\frac{e}{c} \vec{A}\right)^{2}=\left(\vec{p}-\frac{e}{2 c} \vec{B} \times \vec{x}\right)^{2} \approx \vec{p}^{2}-\frac{e}{c} \vec{B} \cdot \vec{L},
$$

where $\vec{L}=\vec{x} \times \vec{p} \quad$ and $\quad \hat{\vec{S}}=\frac{1}{2} \hbar \hat{\hat{\sigma}}$ are the operator of orbital angular momentum and the spin operator respectively. 
So that the Equation (26) finally takes the form of

$$
i \hbar \frac{\partial}{\partial t} \phi_{n c}=\left\{\frac{\vec{p}^{2}}{2 m_{0}-\frac{e}{\hbar c^{2}} \Xi_{\Theta}}-\frac{e}{c\left(2 m_{0}-\frac{e}{\hbar c^{2}} \Xi_{\Theta}\right)}(\vec{L}+2 \hat{\vec{S}}) \vec{B}+e A_{0}+\frac{e}{\hbar} \Xi_{\Theta}\right\} \phi_{n c} .
$$

While we are in very tiny space scales, so the NC term $\Xi_{\Theta} \ll 1$, it is possible to use the Maclaurin series, by changing the variable

$$
\begin{gathered}
\frac{e}{2 m_{0} \hbar c^{2}} \Xi_{\Theta}=\Omega_{\Theta}, \\
\frac{e}{2 m_{0}\left(1-\frac{e}{2 m_{0} \hbar c^{2}} \Xi_{\Theta}\right)} \cong \frac{1}{2 m_{0}} \sum_{j=0}^{n} \Omega_{\Theta}^{j},
\end{gathered}
$$

we find that the Equation (28) goes to

$$
i \hbar \frac{\partial}{\partial t} \phi_{n c}=\left\{\frac{1}{2 m_{0}} \sum_{j=0}^{n} \Omega_{\Theta}^{j}\left(\vec{p}^{2}-\frac{e}{c}(\vec{L}+2 \hat{\vec{S}}) \vec{B}\right)+e A_{0}+2 m_{0} c^{2} \Omega_{\Theta}\right\} \phi_{n c} .
$$

The Equation (28) represents the phase-space noncommutative SchrödingerPauli equation, and it contains the NC kinetic energy operator and the NC Zeeman coupling term (which had been added by hand by Pauli when we talk about the commutative term), and the term that associated with the NC diamagnetism, in the absence of magnetic field $(A=B=0)$, the Equation (31) takes its original form without the information about the spin, which is the noncommutative Schrödinger equation as follows

$$
i \hbar \frac{\partial}{\partial t} \phi_{n c}=\left\{\frac{1}{2 m_{0}} \sum_{j=0}^{n} \Omega_{\Theta}^{j}\left(\vec{p}^{2}\right)+2 m_{0} c^{2} \Omega_{\Theta}\right\} \phi_{n c} .
$$

The Equation (31) is a first order equation of $\frac{1}{m}$, the nonrelativistic expansion of this equation allows to add potentials such the electrical potential, but also to find corrections terms if one realize the development in the second and third order of $\frac{1}{m}$, precisely we predict that in the seconde order we find the Darwin interaction and Spin-Orbit coupling NC terms knowing that Darwin's $\mathrm{NC}$ term is interpreted as a correction of the potential energy due to the Zitterbewegung phenomenon (the tremor movement) [32] [33], in the third order we find corrections of the kinetic energy and temporal dependence of the electric field NC terms.

\section{Conclusion}

In conclusion, the nonrelativistic limit of the Dirac equation with electromagnetic potential has been studied in noncommutative phase-space using the large and small wave-function components approach. We find that the effect of the noncommutativity in phase on the nonrelativistic limit vanished, but the effect of the 
noncommutativity in space appeared widely and it reduced in the NC term $\Xi_{\Theta}$ (at least to the order of approximation we have considered). Under the condition that space-space and momentum-momentum are all commutative (namely, $\Theta=\bar{\Theta}=0$ ), the results return to that of the usual quantum mechanics.

\section{Acknowledgements}

The author would like to thank Pr Lyazid Chetouani for the interesting comments and suggestions.

\section{Conflicts of Interest}

The authors declare no conflicts of interest regarding the publication of this paper.

\section{References}

[1] Snyder, H.S. (1946) Quantized Space-Time. Physical Review, 71, 38. https://doi.org/10.1103/PhysRev.71.38

[2] Yang, C.N. (1947) On Quantized Space-Time. Physical Review, 72, 874. https://doi.org/10.1103/PhysRev.72.874

[3] Banks, T., Fischler, W., Shenker, S. and Susskind, L. (1997) $M$ Theory as a Matrix Model: A Conjecture. Physical Review D, 55, 5112.

https://doi.org/10.1103/PhysRevD.55.5112

[4] Douglas, M.R. and Nekrasov, N.A. (2001) Noncommutative Field Theory. Reviews of Modern Physics, 73, 977-1029. https://doi.org/10.1103/RevModPhys.73.977

[5] Connes, A., Douglas, M.R. and Schwarz, A. (1998) Noncommutative Geometry and Matrix Theory. Journal of High Energy Physics, 9802, 003. https://doi.org/10.1088/1126-6708/1998/02/003

[6] Seiberg, N. and Witten, E. (1999) String Theory and Noncommutative Geometry. Journal of High Energy Physics, 9909, 032. https://doi.org/10.1088/1126-6708/1999/09/032

[7] Chaichian, M., Demichev, A. and Presnajder, P. (2000) Quantum Field Theory on Non-Commutative Space-Times and the Persistence of Ultraviolet Divergences. Nuclear Physics B, 567, 360. https://doi.org/10.1016/S0550-3213(99)00664-1

[8] Duffin, R.J. (1938) On the Characteristic Matrices of Covariant Systems. Physical Review, 54, 1114. https://doi.org/10.1103/PhysRev.54.1114

[9] Kemmer, N. (1938) Quantum Theory of Einstein-Bose Particles and Nuclear Interaction. Proceedings of the Royal Society A, 166, 127. https://doi.org/10.1098/rspa.1938.0084

[10] Kemmer, N. (1939) The Particle Aspect of Meson Theory. Proceedings of the Royal Society $A, 173,91$. https://doi.org/10.1098/rspa.1939.0131

[11] Foldy, L. and Wouthuysen, S. (1950) On the Dirac Theory of Spin 1/2 Particles and Its Non-Relativistic Limit. Physical Review, 78, 29.

[12] Nikitin, A.G. (1998) On Exact Foldy-Wouthuysen Transformation. Journal of Physics A: Mathematical and General, 31, 3297-3300. https://doi.org/10.1088/0305-4470/31/14/015

[13] Jansen, G. and Hess, B.A. (1989) Revision of the Douglas-Kroll Transformation. Physical Review A, 39, 6016-6017. https://doi.org/10.1103/PhysRevA.39.6016 
[14] Reiher, M. (2006) Douglas-Kroll-Hess Theory: A Relativistic Electrons-Only Theory for Chemistry. Theoretical Chemistry Accounts, 116, 241-252.

[15] Nakajima, T. (2012) The Douglas-Kroll-Hess Approach. Chemical Reviews, 112, 385-402. https://doi.org/10.1021/cr200040s

[16] Greiner, W. (1994) Quantum Mechanics. 3rd Edition, Springer, Berlin, Heidelberg.

[17] Bechouche, P., Mauser, N. and Bechouche, P. (1998) (Semi)-Nonrelativistic Limits of the Dirac Equation with External Time-Dependent Electromagnetic Field. Communications in Mathematical Physics, 197, 405-425. https://doi.org/10.1007/s002200050457

[18] Bjorken, J.D. and Drell, S.D. (1964) Relativistic Quantum Mechanics. McGraw-Hill, New York.

[19] Davydov, A.S. (1965) Quantum Mechanics. 2nd Edition, Pergamon, Oxford, 63.

[20] Messiah, A. (1968) Quantum Mechanics, Vol. II. Wiley, New York, 4. V.B.

[21] Berestetskii, V.B., Lifshitz, E.M. and Pitaevskii, L.P. (1989) Quantum Electrodynamics. 2nd Edition, Pergamon, Oxford.

[22] Jiang, X., Long, C. and Qin, S. (2013) Solution of Dirac Equation with the Time-Dependent Linear Potential in Non-Commutative Phase. Journal of Modern Physics, 4, 940-944. https://doi.org/10.4236/jmp.2013.47126

[23] Hassenabadi, H., Molaee, Z. and Zarrinkamar, S. (2014) Noncommutative Phase Space Schrödinger Equation with Minimal Length. Advances in High Energy Physics, 2014, Article ID: 459345.

[24] Mirza, B. and Mohadesi, M. (2014) The Klein-Gordon and the Dirac Oscillators in a Noncom-Mutative Space. Theor. Phys. Communications in Theoretical Physics, 42, 664-668.

[25] Hua, W.J. and Kang, L. (2007) He-McKellar-Wilkens Effect in Noncommutative Space. Chinese Physics Letters, 24, 5. https://doi.org/10.1088/0256-307X/24/1/002

[26] Kupriyanov, V.G. (2013) Hydrogen Atom on Curved Noncommutative Space. Journal of Physics A, 46, Article ID: 245303. https://doi.org/10.1088/1751-8113/46/24/245303

[27] Adorno, T.C., Baldiotti, M.C., Chaichian, M., Gitman, D.M. and Tureanu, A. (2009) Dirac Equation in Noncommutative Space for Hydrogen Atom. Physics Letters B, 682, 235. https://doi.org/10.1016/j.physletb.2009.11.003

[28] Bertolamia, O. and Queirozb, R. (2011) Phase-Space Noncommutativity and the Dirac Equation. Physics Letters A, 375, 4116-4119. https://doi.org/10.1016/j.physleta.2011.09.053

[29] Gosselin, P., Bérard, A. and Mohrbach, H. (2006) Semiclassical Diagonalization of Quantum Hamiltonian and Equations of Motion with Berry Phase Corrections. The European Physical Journal B, 58, 137-148. https://doi.org/10.1140/epjb/e2007-00212-6

[30] Nowakowski, M. (1999) The Quantum Mechanical Current of the Pauli Equation. American Journal of Physics, 67, 916. https://doi.org/10.1119/1.19149

[31] Torres del Castillo, G.F. and Velázquez Castro, J. (2004) Schrödinger-Pauli Equation for Spin-3/2 Particles. Revistamexicana de física, 50, 306-310.

[32] Hestenes, D. (1990) The Zitterbewegung Interpretation of Quantum Mechanics. Foundations of Physics, 20, 1213-1232. https://doi.org/10.1007/BF01889466

[33] Huang, K. (1952) On the Zitterbewegung of the Dirac Electron. American Journal of Physics, 20, 479. https://doi.org/10.1119/1.1933296 


\section{Appendix}

Calculations between moving from the Equation (13) to the Equation (14):

Using $\bar{\Theta}_{i j}=\bar{\Theta} \epsilon_{i j}$ and $\bar{\Theta}_{k}=\frac{1}{2} \epsilon_{k i j} \bar{\Theta}_{i j}$ then

$c \alpha_{i} \frac{1}{2 \hbar} \bar{\Theta}_{i j} x_{j}=2 c \frac{1}{2 \hbar} \bar{\Theta}_{k} \epsilon_{k i j}^{-1} \alpha_{i} x_{j}=c \frac{1}{2} \bar{\Theta}_{k} \epsilon_{k i j} \alpha_{i} x_{j}$, knowing that $\epsilon_{k i j}=\epsilon_{i j k}$,

we know that $(u \times v)_{\mu}=\epsilon_{\mu \nu \lambda} u_{v} \cdot v_{\lambda}$, so that

$$
c \frac{1}{2} \bar{\Theta}_{k} \epsilon_{k i j} \alpha_{i} x_{j}=\frac{c}{\hbar}(\vec{\alpha} \times \vec{x})_{k} \cdot \bar{\Theta}_{k}=\frac{c}{\hbar}(\vec{\alpha} \times \vec{x}) \cdot \overrightarrow{\bar{\Theta}},
$$

with the same manner we prove that

$$
\begin{aligned}
-i e \Theta_{k} \epsilon_{a b k} \partial_{a}\left(\alpha_{i} A_{i}-A_{0}\right) \partial_{b} & =\frac{-i e \hbar}{\hbar} \Theta_{k} \epsilon_{a b k} \partial_{a}\left(\vec{\alpha} \vec{A}-A_{0}\right) \partial_{b} \\
& =\frac{e}{\hbar}\left(\vec{\nabla}\left(\vec{\alpha} \vec{A}-A_{0}\right) \times \vec{p}\right) \cdot \vec{\Theta}
\end{aligned}
$$

